\title{
Effects of Anxiety Sensitivity and Hearing Loss on Tinnitus Symptom Severity
}

\author{
Kyung Ray Moon', Subin Park², YouJi Jung ${ }^{3}$, AhReum Lee², and Jung Hyun Lee ${ }^{4}$ \\ ${ }^{1}$ Tinnitus Clinic, Soree Ear Hospital, Seoul, Republic of Korea \\ ${ }^{2}$ Department of Research Planning, Mental Health Research Institute, National Center for Mental Health, Seoul, Republic of Korea \\ ${ }^{3}$ Department of Clinical Research, Mental Health Research Institute, National Center for Mental Health, Seoul, Republic of Korea \\ ${ }^{4}$ Department of Psychiatry, National Center for Mental Health, Seoul, Republic of Korea
}

Objective The aim of the present study was to examine the relative role of anxiety sensitivity and hearing loss on the tinnitus symptoms severity in a large clinical sample of patients with tinnitus.

Methods A total of 1,705 patients with tinnitus who visited the tinnitus clinic underwent the pure-tone audiometric testing and a battery of self-report questionnaires. Multiple linear regression analyses were performed to identify the relationship of anxiety sensitivity and hearing loss to tinnitus symptoms severity.

Results Both anxiety sensitivity and hearing loss were a significant association with of annoyance (anxiety sensitivity $\beta=0.11, \mathrm{p}=0.010$; hearing loss $\beta=0.09, \mathrm{p}=0.005$ ) and THI score (anxiety sensitivity $\beta=0.21, \mathrm{p}<0.001$; hearing loss $\beta=0.10, \mathrm{p}<0.001$ ) after adjusting for confounding factors. Meanwhile, the awareness time $(\beta=0.19, \mathrm{p}<0.001)$ and loudness $(\beta=0.11, \mathrm{p}<0.001)$ of tinnitus was associated with only the hearing loss but not with anxiety sensitivity

Conclusion Our results indicate that both hearing loss and anxiety sensitivity were associated with increased tinnitus symptom severity. Furthermore, these associations could be different according to the characteristics of tinnitus symptoms.

Psychiatry Investig 2018;15(1):34-40

Key Words Anxiety sensitivity, Anxiety sensitivity index, Hearing loss, Tinnitus, Tinnitus handicap inventory.

\section{INTRODUCTION}

Tinnitus refers to the false sensation of a ringing or buzzing sound in the absence of external stimuli. ${ }^{1}$ The prevalence of tinnitus is $10-15 \%$ in the general population, ${ }^{2}$ with approximately one third of individuals with tinnitus experiencing distress and reduced quality of life. ${ }^{3}$ Tinnitus symptoms are often attributed to diverse causes, including hearing loss, $\mathrm{Me}$ niere's disease, and otitis media; however, in many cases, no cause is found. ${ }^{4}$ As subjective tinnitus generally involves complex symptoms, it is not always possible to fully eliminate. ${ }^{4,5}$ The clinical characteristics of tinnitus (e.g., duration of aware-

Received: January 10, 2017 Revised: February 26, 2017

Accepted: March 9, 2017 Available online: November 16, 2017

$\triangle$ Correspondence: Jung Hyun Lee, MD, PhD

Department of Psychiatry, National Center for Mental Health, 12 Yongmasanro, Gwangjin-gu, Seoul 04933, Republic of Korea

Tel: +82-2-2204-0136, Fax: +82-2-2204-0280, E-mail: leejunghyun1@gmail.com

(c) This is an Open Access article distributed under the terms of the Creative Commons Attribution Non-Commercial License (http://creativecommons.org/licenses/by$\mathrm{nc} / 4.0$ ) which permits unrestricted non-commercial use, distribution, and reproduction in any medium, provided the original work is properly cited. ness, loudness, location) can vary widely, ${ }^{4}$ as can symptom severity, ranging from barely noticeable to unbearable and debilitating. ${ }^{4}$ Tinnitus is believed to have a complex etiology, including ontological and neuropsychological factors ${ }^{6}$; however, due to the subjectivity of its symptoms, what contributes to tinnitus symptom severity is poorly understood.

Hearing impairment is one of the auditory factors frequently accompanying tinnitus. Several epidemiological studies have shown that the occurrence of tinnitus is related to hearing impairment. ${ }^{78}$ However, not all (approximately 30\%) of patients with hearing loss exhibit tinnitus symptoms. ${ }^{4,9} \mathrm{Neu}-$ roanatomical research has considered the role of neural plasticity followed by reorganization of the cerebral cortex as a cause of tinnitus. ${ }^{10}$ Hearing loss can lead to a reorganization of the central auditory system. ${ }^{10}$ However, only a few studies with small samples have used objective audiometric tests to examine hearing levels in patients with tinnitus. ${ }^{7.8}$ Therefore, despite its clinical importance, the relationship between hearing loss and tinnitus symptom severity remains unclear.

Other important etiologies of tinnitus are neuropsychologi- 
cal factors such as depression and anxiety. ${ }^{11,12}$ Several studies have described tinnitus as a subjective experience associated with emotional and cognitive problems rather than objective auditory problems. ${ }^{11,13}$ Additionally, the high prevalence of psychological symptoms, including depression and anxiety, in patients with tinnitus is well documented. ${ }^{14-17}$ Despite the strong correlations between tinnitus and psychiatric symptoms, ${ }^{17}$ a causal relationship has not been established because depressive and anxiety symptoms reflect a state, rather than a trait, before illness. Several reports have suggested that personality or trait factors such as neuroticism, ${ }^{18}$ temperament, ${ }^{19}$ and trait anxiety ${ }^{20,21}$ might exacerbate tinnitus symptoms. Anxiety sensitivity is also considered a premorbid personal characteristic, defined as a tendency toward anxious responses to somatic perception. ${ }^{22}$ Several studies have shown that anxiety sensitivity is associated with tinnitus symptoms. ${ }^{23,24}$ Andersson and Vretblad. ${ }^{23}$ suggested that anxiety sensitivity could be a causative factor for tinnitus-related distress. However, further research is required to evaluate the role of anxiety sensitivity as a predictor of tinnitus symptom severity in a large sample.

We presume that both hearing loss (i.e., reorganization of auditory nerves) and anxiety sensitivity (susceptibility to somatic sensations) are associated with tinnitus symptoms. Accordingly, these two factors must be considered important predictors of tinnitus symptom severity. The aim of the current study was determine the precise effects of anxiety sensitivity and hearing loss on tinnitus symptom severity in a large clinical sample of patients with tinnitus. Furthermore, we hypothesized that these associations differ according to the characteristics of tinnitus symptoms.

\section{METHODS}

\section{Participants and procedure}

We reviewed the medical records and self-reported questionnaire responses of 1,797 patients who presented with tinnitus symptoms as a chief complaint at the tinnitus clinic in Soree Ear Hospital between May 2015 and April 2016. Basic demographic data, self-report questionnaires and pure-tone audiometry results were obtained from patients' medical records. Patients underwent audiometry and completed a battery of self-report questionnaires during waiting period before clinic encounter. The Korean versions of the Tinnitus Handicap Inventory (THI), Hospital Anxiety and Depression Scale (HADS), and Anxiety Sensitivity Index (ASI), and visual analog scales (VASs) were used to examine tinnitus symptoms and psychological status as a part of medical care. This study was reviewed by the Institutional Review Board (IRB) at the National Center for Mental Health and was considered exempt from informed consent requirements (2015-04-13).

\section{Measures}

Information regarding patients' tinnitus-related symptoms (i.e., awareness time, loudness, degree of annoyance, and impact on life) over the preceding month was assessed using VASs. Awareness time of tinnitus was measured as the proportion of the day during which patients were aware of their tinnitus symptoms, with responses ranging from $0 \%$ (no awareness during the day) to $100 \%$ (full awareness all day). Responses regarding loudness of tinnitus ranged from 0 (inaudible) to 10 (intolerably loud). Degree of annoyance ranged from 0 (no annoyance) to 10 (extreme annoyance). Finally, the impact of tinnitus on life ranged from 0 (no impact) to 10 (incapacitation).

The Korean version of the THI was used to measure the severity of tinnitus-related handicap. ${ }^{25}$ This scale was originally developed by Newman et al. to measure the perceived disability caused by tinnitus and related distress. The Korean version of the THI was standardized by Kim et al. ${ }^{26}$ The questionnaire comprises 25 items that determine whether participants experience particular difficulties (e.g., difficulty concentrating, confusion, and anger) resulting from tinnitus. Possible responses include "yes," "sometimes", and "no" (yes= 4 points, sometimes $=2$ points, no $=0$ points). Total scores range from 0 to 100, with higher scores indicating higher levels of perceived tinnitus-related handicap and distress. ${ }^{25,26}$

The $\mathrm{ASI}^{22,27}$ was used to measure fear of anxiety-related sensations and the belief that anxiety symptoms have negative consequences. The questionnaire comprises 16 items measuring the extent to which participants are concerned about the negative consequences of anxiety symptoms. ${ }^{22}$ Responses are provided using a five-point Likert scale ranging from 0 (very little) to 4 (very much). The total ASI score ranges from 0 to 64 and represents an individual's level of anxiety sensitivity. ${ }^{27}$ Note that the ASI score reflects responses to physical symptoms associated with anxiety rather than individuals' current levels of anxiety. ${ }^{22}$ Furthermore, anxiety sensitivity has been shown to be a comparatively stable characteristic. ${ }^{28,29}$ The cutoff for high ASI was set at 21 or greater.

The HADS, ${ }^{30,31}$ a measure of current anxiety and depressive symptoms among patients visiting general hospitals, was used to assess participants' anxiety and depression levels. The scale comprises 14 items divided between two subscales measuring anxiety and depressive symptoms, each with a cutoff score of 8 for clinical levels of anxiety/depression. . $^{30,31}$

Pure-tone audiometric testing was performed in soundtreated booths (Sontek Company, Paju, Korea) using 2-channel pure-tone audiometers (Madsen Auricla/Astera2, Otometrics) equipped with TDH-49 earphones. Pure-tone air conduction thresholds were obtained for each ear at 500, 1,000, 
2,000, and 4,000 Hz. Hearing loss was considered present if subjects' pure-tone average exceeded $25 \mathrm{~dB}$. We also graded the severity of participants' hearing loss as follows: mild (25$40 \mathrm{~dB})$, moderate $(40-60 \mathrm{~dB})$, and severe (>60 dB).

\section{Data analysis}

Analyses were performed using SPSS Statistics 21.0 for Windows (IBM Corp., Armonk, NY, USA). We compared the differences in demographic and clinical variables between four groups based on anxiety sensitivity level (AS) and severity of hearing loss (HL), including those with normal anxiety sensitivity and hearing level (AS-/NH), normal anxiety sensitivity and hearing loss (AS/-HL), high anxiety sensitivity and normal hearing $(\mathrm{AS}+/ \mathrm{NH})$, and high anxiety sensitivity and hearing loss (AS+/HL). Comparisons were performed via analysis of variance (ANOVA) with Tukey's HSD post-hoc tests for continuous variables and Kruskal-Wallis $\mathrm{H}$ tests for categorical variables. To determine which variables (hearing level, anxiety sensitivity, current anxiety, and depressive symptoms) predict tinnitus symptom severity (awareness, loudness, annoyance, impact on life, and THI score), we per- formed multiple linear regression analyses after controlling for age, sex, duration of illness, and location of tinnitus. The significance level was set at $\mathrm{p}<0.05$. We adjusted the $\mathrm{p}$-values for each regression model for multiple comparisons using Bonferroni corrections.

\section{RESULTS}

\section{Description of study population and the result of group comparisons}

Participants' sociodemographic and clinical characteristics are summarized in Table 1 . Of the 1,705 patients with tinnitus included in this study, 749 (43.8\%) were men. Participants' mean age was $53.48 \pm 14.47$ (range: $18^{-97}$ ) years, and the mean tinnitus duration was $38.71 \pm 73.80$ (range: 0-696) months. Regarding the perceived tinnitus location, 470 (27.6\%) patients reported it in their right ear, $543(31.8 \%)$ in their left ear, 541 (31.7\%) in both ears, 74 (4.3\%) inside the head and 49 (2.9\%) in other locations. The missing value was 28 (1.6\%). The average proportion of the day during which participants were aware of tinnitus symptoms was $67.82 \pm 31.75 \%$. The mean

Table 1. Group comparisons of demographic and clinical variables among patients with tinnitus $(\mathrm{N}=1,705)$

\begin{tabular}{|c|c|c|c|c|c|c|}
\hline Variables & $\begin{array}{l}\text { All participants } \\
\qquad \mathrm{N}=1,705\end{array}$ & $\begin{array}{l}\text { AS-/NH } \\
\mathrm{N}=306\end{array}$ & $\begin{array}{l}\text { AS-/HL } \\
\mathrm{N}=1,000\end{array}$ & $\begin{array}{c}\mathrm{AS}+/ \mathrm{NH} \\
\mathrm{N}=84\end{array}$ & $\begin{array}{c}\mathrm{AS}+/ \mathrm{HL} \\
\mathrm{N}=315\end{array}$ & Statistics \\
\hline Age (year) & $53.48 \pm 14.47$ & $49.75 \pm 15.32$ & $54.50 \pm 14.00^{*}$ & $48.32 \pm 14.74^{* *}$ & $55.26 \pm 14.15^{* * * * *}$ & $\mathrm{~F}=13.90, \mathrm{p}<0.001$ \\
\hline Gender (\%) & & & & & & $\chi^{2}=18.65, p<0.001$ \\
\hline Men & $746(43.8)$ & $115(37.6)$ & $481(48.1)$ & $31(36.9)$ & $119(37.8)$ & \\
\hline Women & $954(56.0)$ & $190(62.1)$ & $516(51.6)$ & $53(63.1)$ & $195(61.9)$ & \\
\hline Duration of illness (month) & $38.71 \pm 73.80$ & $22.60 \pm 50.22$ & $42.32 \pm 77.10^{*}$ & $11.74 \pm 21.35^{* *}$ & $50.08 \pm 86.36^{* * * * *}$ & $\mathrm{~F}=12.13, \mathrm{p}<0.001$ \\
\hline Location of tinnitus (\%) & & & & & & $\chi^{2}=27.48, p<0.001$ \\
\hline Right ear & $470(27.6)$ & $109(35.6)$ & $261(26.1)$ & $30(35.7)$ & $70(22.2)$ & \\
\hline Left ear & $543(31.8)$ & $107(35.0)$ & $313(31.3)$ & $28(33.3)$ & $95(30.2)$ & \\
\hline Both ears & $541(31.7)$ & $75(24.5)$ & $331(33.1)$ & $21(25.0)$ & $114(36.2)$ & \\
\hline Inside head & $74(4.3)$ & $9(2.9)$ & $47(4.7)$ & $1(1.2)$ & $17(5.4)$ & \\
\hline Others & $49(2.9)$ & $4(1.3)$ & $32(3.2)$ & $4(4.8)$ & $9(2.9)$ & \\
\hline \multicolumn{7}{|l|}{ Tinnitus-related characteristics } \\
\hline Awareness & $67.82 \pm 31.75$ & $57.48 \pm 32.67$ & $69.00 \pm 31.26^{*}$ & $65.61 \pm 29.93$ & $75.43 \pm 30.12^{* * * *}$ & $\mathrm{~F}=16.88, \mathrm{p}<0.001$ \\
\hline Loudness & $5.23 \pm 2.92$ & $4.47 \pm 2.31$ & $5.17 \pm 3.20^{*}$ & $5.45 \pm 2.32^{*}$ & $6.16 \pm 2.41^{* * * *}$ & $\mathrm{~F}=17.22, \mathrm{p}<0.001$ \\
\hline Annoyance & $5.51 \pm 2.70$ & $4.87 \pm 2.55$ & $5.21 \pm 2.61$ & $6.11 \pm 2.63^{* * * *}$ & $6.99 \pm 2.59 *, * *, * * *$ & $\mathrm{~F}=42.28, \mathrm{p}<0.001$ \\
\hline Impact of on life & $4.51 \pm 3.53$ & $3.97 \pm 2.42$ & $4.25 \pm 4.03$ & $5.45 \pm 2.64^{*, * *}$ & $5.67 \pm 2.66^{* * * *}$ & $\mathrm{~F}=16.43, \mathrm{p}<0.001$ \\
\hline THI score & $37.24 \pm 25.59$ & $29.84 \pm 21.41$ & $32.48 \pm 23.46$ & $51.93 \pm 26.29^{* * * *}$ & $55.59 \pm 25.61^{* * * *}$ & $\mathrm{~F}=97.32, \mathrm{p}<0.001$ \\
\hline \multicolumn{7}{|l|}{ Psychiatric characteristics } \\
\hline HADS-anxiety & $5.79 \pm 4.07$ & $4.74 \pm 3.00$ & $4.66 \pm 3.28$ & $9.57 \pm 4.62^{* * * *}$ & $9.42 \pm 4.35^{* * * *}$ & $\mathrm{~F}=184.31, \mathrm{p}<0.001$ \\
\hline HADS-depression & $6.14 \pm 3.94$ & $5.30 \pm 3.46$ & $5.29 \pm 3.48$ & $9.15 \pm 4.20^{* * * *}$ & $8.87 \pm 4.08^{* * * *}$ & $\mathrm{~F}=101.05, \mathrm{p}<0.001$ \\
\hline
\end{tabular}

All participants were divided into four groups including normal anxiety sensitivity and hearing level (AS-/NL), normal anxiety sensitivity and hearing loss (AS/-HL), high anxiety sensitivity and normal hearing (AS+/NH), and high anxiety sensitivity and hearing loss (AS+/HL). Hearing loss was considered if pure-tone average $>25 \mathrm{~dB}$. The ASI cutoff for high anxiety sensitivity set at $>21$. ${ }^{*} \mathrm{p}<0.05 \mathrm{vs}$. AS-/NH, ${ }^{* *} \mathrm{p}<0.05 \mathrm{vs}$. AS-/HL, ${ }^{* * *}$ p $<0.05$ vs. AS+/NL. THI: Tinnitus Handicap Inventory, ASI: Anxiety Sensitivity Index, HADS: Hospital Anxiety Depression Scale 
scores for tinnitus loudness, degree of annoyance, and impact on life were $5.23 \pm 2.92,5.51 \pm 2.70$, and $4.51 \pm 3.53$, respectively. The mean total THI score was $37.24 \pm 25.59$. Furthermore, the mean total ASI score was $13.56 \pm 12.47$, and the numbers of participants whose anxiety sensitivity levels were categorized as normal and high according to the abovementioned cutoff were 1,306 (76.6\%) and 399 (23.4\%), respectively. The number of participants with normal hearing was 390 (22.9\%). Of the patients with hearing loss, 308 (18.1\%), 342 (20.1\%), and 665 (39.0\%) exhibited mild, moderate, and severe hearing loss, respectively.

Table 1 also shows results of the comparison of the four groups. There were 306 (17.9\%) participants in the AS-/NH group, 1,000 (55.6\%) in the AS-/HL group, 84 (4.9\%) in the AS+/NH group, and 315 (18.5\%) in the AS+/HL group. We observed significant differences in tinnitus symptom severity between these groups [awareness: $\mathrm{F}(3,1525)=16.88, \mathrm{p}<0.001$; loudness: $\mathrm{F}(3,1556)=17.22, \mathrm{p}<0.001$; annoyance: $\mathrm{F}(3,1565)=$ 42.28, $\mathrm{p}<0.001$; impact on life: $\mathrm{F}(3,1557)=16.43$, $\mathrm{p}<0.003$; THI: $\mathrm{F}(3,1701)=97.32, \mathrm{p}<0.001]$. The results of the post-hoc analysis of group differences are shown in Table 1, Figure 1.

\section{Multiple regression analyses}

Table 2 depicts the results of the multiple linear regression
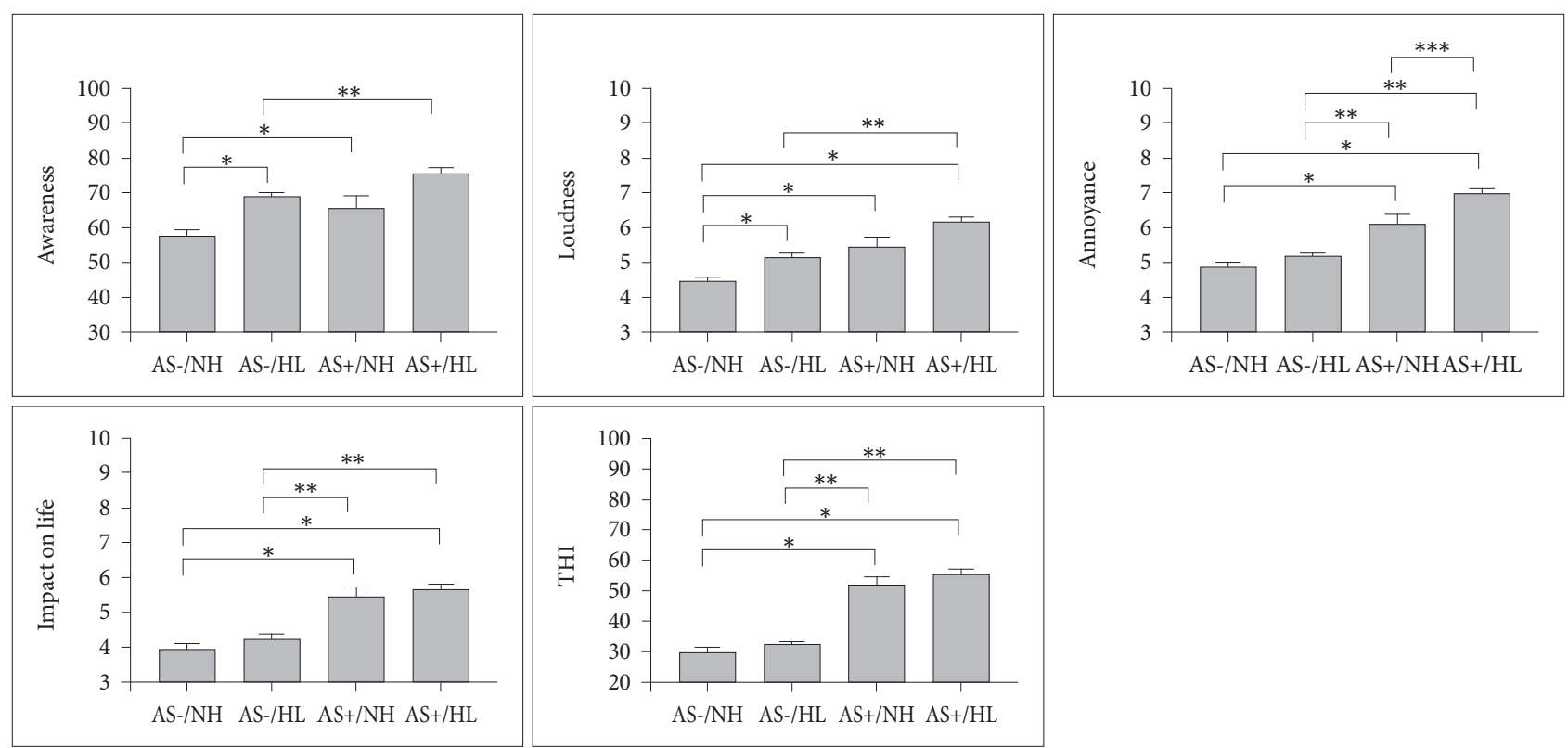

Figure 1. Mean \pm SE of one-way ANOVA with Tukey's HSD post hoc test. Mean values of tinnitus symptoms were compared between four groups based on anxiety sensitivity level (AS) and severity of hearing loss (HL), including those with normal anxiety sensitivity and hearing level (AS-/NH), normal anxiety sensitivity and hearing loss (AS/-HL), high anxiety sensitivity and normal hearing $(\mathrm{AS}+/ \mathrm{NH})$, and high anxiety sensitivity and hearing loss $(\mathrm{AS}+/ \mathrm{HL})$. Symbols represent a significant difference $(p<0.05)$ compared with the AS-/NH group $(*)$, the AS-/HL group $(* *)$, and the AS+/NH group $\left({ }^{* *}\right)$.

Table 2. Multiple linear regression models of the relations between tinnitus symptoms severity and clinical variables

\begin{tabular}{|c|c|c|c|c|c|c|c|c|c|c|}
\hline \multirow{2}{*}{ Variables } & \multicolumn{2}{|c|}{ Awareness } & \multicolumn{2}{|c|}{ Loudness } & \multicolumn{2}{|c|}{ Annoyance } & \multicolumn{2}{|c|}{ Impact on life } & \multicolumn{2}{|c|}{ THI score } \\
\hline & $\beta$ & $\mathrm{t}$ & $\beta$ & $\mathrm{t}$ & $\beta$ & $\mathrm{t}$ & $\beta$ & $t$ & $\beta$ & $\mathrm{t}$ \\
\hline Age & $0.09^{*}$ & 3.13 & 0.07 & 2.50 & 0.02 & 0.84 & 0.00 & 0.10 & 0.03 & 1.18 \\
\hline Gender & -0.05 & -1.79 & 0.03 & 0.98 & 0.00 & 0.05 & 0.01 & 0.45 & 0.04 & 1.60 \\
\hline Duration of illness (month) & $0.12^{* *}$ & 4.23 & $0.10^{* *}$ & 3.52 & 0.06 & 2.25 & $0.10^{* *}$ & 3.29 & $0.07^{*}$ & 3.10 \\
\hline Location of Tinnitus & 0.07 & 2.32 & 0.00 & 0.11 & -0.03 & -1.08 & -0.01 & -0.22 & $-0.06^{*}$ & -2.87 \\
\hline HADS-anxiety score & 0.04 & 0.84 & $0.14^{* *}$ & 3.22 & $0.30^{* *}$ & 7.40 & $0.15^{* *}$ & 3.51 & $0.32^{* *}$ & 9.34 \\
\hline HADS-depression score & 0.03 & 0.72 & 0.04 & 1.01 & 0.05 & 1.39 & 0.07 & 1.76 & $0.14^{* *}$ & 4.44 \\
\hline ASI total score & 0.05 & 1.26 & 0.05 & 1.32 & $0.11^{*}$ & $3.18^{*}$ & 0.08 & 2.17 & $0.21^{* *}$ & 7.15 \\
\hline Severity of hearing loss ${ }^{\dagger}$ & $0.19^{* *}$ & 6.23 & $0.11^{* *}$ & 3.60 & $0.09^{* *}$ & 3.33 & 0.03 & 0.93 & $0.10^{* *}$ & 4.27 \\
\hline
\end{tabular}

$\mathrm{R}^{2}$ is 0.101 for the awareness. 0.084 for the loudness, 0.199 for the annoyance, 0.085 for the impact on life and 0.379 for THI score. Bonferroni corrected $\mathrm{p}$ values ${ }^{*}<0.05,{ }^{* *}<0.01{ }^{\dagger}{ }^{\dagger}$ severity of hearing loss: normal $(<25 \mathrm{~dB})$, mild $(25-40 \mathrm{~dB})$, moderate $(40-60 \mathrm{~dB})$, and severe $(>60 \mathrm{~dB})$. HADS: Hospital Anxiety Depression Scale, ASI: Anxiety Sensitivity Index, THI: Tinnitus Handicap Inventory 
analyses. Among the independent variables (e.g., severity of hearing loss, ASI score), severity of hearing loss was the only predictor of awareness of tinnitus $(\beta=0.19, \mathrm{t}=6.23, \mathrm{p}<0.001)$ and loudness $(\beta=0.11, t=3.60, p<0.001)$. Annoyance and THI score were significantly predicted by both ASI score (annoyance: $\beta=0.11, t=3.18, p=0.010$; THI score: $\beta=0.21, t=7.15, p<$ 0.001 ) and severity of hearing loss (annoyance: $\beta=0.09, t=3.33$, $\mathrm{p}=0.005$; THI score: $\beta=0.10, \mathrm{t}=4.27, \mathrm{p}<0.001$ ). Neither predictor had a significant relationship with impact on life.

\section{DISCUSSION}

Our study investigated the effects of anxiety sensitivity and severity of hearing loss on tinnitus symptom severity. In the group comparison, the AS+/HL group showed the most severe symptoms for each measure of tinnitus severity, including greater awareness, louder sound, greater annoyance level, worse impact on life, and higher THI scores than did the AS-/NH group. Furthermore, the groups with high anxiety sensitivity (AS+/NH and AS+/HL) showed more severe symptoms, including greater annoyance, impact on life, and THI scores, irrespective of their degree of hearing loss (i.e., compared to the AS-/NH and AS-/HL). Among the groups with low anxiety sensitivity, awareness and loudness of tinnitus were significantly greater among patients with hearing loss (AS-/HL) than among those with normal hearing (AS-/NH). Finally, the results of the multiple regression analysis showed that severe hearing impairment and high levels of anxiety sensitivity are significant predictors of tinnitus symptom severity, although the precise aspects of tinnitus severity to which they are related differ. Specifically, awareness and loudness of tinnitus were significantly related to hearing loss, but not anxiety sensitivity. Conversely, annoyance and tinnitus-related handicap were predicted by higher anxiety sensitivity as well as severity of hearing loss.

To our knowledge, this study examined the largest clinical sample of patients with tinnitus in which hearing levels (measured using pure-tone audiometry) and anxiety sensitivity to date. Due to the multidimensional nature of tinnitus, accurate assessment of tinnitus severity must be made using a variety of measures. In our study, tinnitus symptom severity was assessed in terms of awareness, loudness, degree of annoyance, impact on life, and tinnitus-related handicap. Awareness and loudness of tinnitus were presumed to be acoustical symptoms rather than subjective ones. However, degree of annoyance, impact on life, and tinnitus-related handicap were measures of the non-auditory symptoms of tinnitus, including psychological distress and low quality of life. ${ }^{32,33}$ The current consensus is that tinnitus symptoms have multiple etiologies. ${ }^{34}$ As such, tinnitus might be related to not only dysfunction of the inner ear or auditory nerves but also to cognitive distortions of somatic sensations. ${ }^{6,34}$ Our findings further support the notion that auditory factors (i.e., hearing loss) are associated with the acoustical symptoms (awareness and loudness), whereas psychological traits (anxiety sensitivity) are linked with the non-auditory symptoms (annoyance, impact on life and tinnitus-related handicap).

Although previous studies have reported a significant relationship between tinnitus severity and psychiatric symptoms. ${ }^{14,35}$ they did not establish causality-as such, their results indicated that high levels of anxiety and depression could result in tinnitus or vice versa. Therefore, we examined the effect of anxiety sensitivity, which is considered a premorbid trait, on tinnitus symptom severity. Anxiety sensitivity reflects emotional awareness and susceptibility to anxiety-related somatic symptoms, rather than individuals' current anxiety level, and has been identified as an individual characteristic or trait. ${ }^{22}$ Individuals with high anxiety sensitivity levels could perceive tinnitus as a life-threatening event, and this cognitive distortion might cause severe distress or tinnitus-related disability. Our findings suggested that anxiety sensitivity was predictive of tinnitus symptom severity, particularly degree of annoyance and tinnitus-related handicap. These associations remained significant even after controlling for demographic factors and hearing levels. Interestingly, in contrast to the results regarding hearing loss, anxiety sensitivity was not associated with awareness or loudness of tinnitus. This is likely because, as noted above, anxiety sensitivity is believed to predict subjective distress better than acoustical symptoms such as awareness and loudness of tinnitus. These results in part accord with several studies showing that anxiety-related temperament can influence tinnitus symptom severity. $18,20,21,23$ For example, Salviati et al. ${ }^{19}$ suggested that harm avoidance was associated with tinnitus symptom severity, and that individuals with high levels of harm avoidance tend to evade risky or harmful signals. Additionally, trait anxiety-defined as the tendency to react to situations that cause anxiety-was associated with tinnitus frequency in a sample of Korean patients aged $10-12$ years. ${ }^{20,36}$ However, anxiety sensitivity differs from trait anxiety in that it involves reaction to nonspecific somatic symptoms. ${ }^{22,28}$ In fact, very few previous studies have evaluated the relationship between severity of tinnitus and anxiety sensitivity. ${ }^{23,24,37}$ To our knowledge, this study is the first to establish a stronger link between anxiety sensitivity and subjective distress (e.g., annoyance, impact on life, tinnitusrelated handicap) compared to auditory symptoms (e.g., awareness, loudness). Individuals with high levels of anxiety sensitivity might worry excessively about tinnitus or engage in negative cognitive appraisals of their experience of tinnitus. This implies that patients with high levels of anxiety sensitiv- 
ity could benefit from a therapeutic approach that focuses on treating physical strain and irrational worry using cognitive behavioral or relaxation therapy. Additionally, cognitive behavioral therapy could relieve anxiety and depressive symptoms as well as tinnitus. ${ }^{38,39}$

This study has some limitations. Because it was a cross-sectional study, no causality can be inferred from the findings. In addition, because the data regarding psychiatric symptoms were collected via self-report scales, we could not confirm diagnoses of psychiatric disorders. However, conducting structured psychiatric interviews with all patients with tinnitus would be time consuming in clinical settings. Further, as our sample comprised patients who had visited one otolaryngology clinic for treatment of tinnitus symptoms, it is possible that the proportion of patients with severe tinnitus or hearing loss was excessively high. However, the large sample size could have compensated for this bias to some extent. Finally, caution should be exercised in generalizing the findings to the general population of individuals with tinnitus.

In conclusion, the results indicated that high anxiety sensitivity and severe hearing loss were associated with increased tinnitus symptom severity. Furthermore, anxiety sensitivity was more likely to affect subjective distress and quality of life relative to awareness of tinnitus and symptom loudness. This finding implies that therapeutic approaches focusing on susceptibility to somatic sensation might help to alleviate tinnitus-related distress. Our findings also enhance our understanding of the multidimensional risk factors of tinnitus symptom severity, and thus could be taken into account in the management of patients with tinnitus.

\section{Acknowledgments}

This work was supported by a clinical research grant (no. 2016-09) from the National Center for Mental Health, Republic of Korea.

\section{REFERENCES}

1. Jastreboff PJ. Phantom auditory perception (tinnitus): mechanisms of generation and perception. Neurosci Res 1990;8:221-254.

2. Shargorodsky J, Curhan GC, Farwell WR. Prevalence and Characteristics of Tinnitus among US Adults. Am J Med 2010;123:711-718.

3. Rizzardo R, Savastano M, Maron MB, Mangialaio M, Salvadori L. Psychological distress in patients with tinnitus. J Otolaryngol 1998;27:21-25.

4. Lockwood AH, Salvi RJ Burkard RF. Tinnitus. N Engl J Med 2002;347: 904-910.

5. Ahmad N, Seidman M. Tinnitus in the older adult: epidemiology, pathophysiology and treatment options. Drugs Aging 2004;21:297-305.

6. Moller AR. Tinnitus: presence and future. Prog Brain Res 2007;166:3-16.

7. Konig O, Schaette R, Kempter R, Gross M. Course of hearing loss and occurrence of tinnitus. Hear Res 2006;221:59-64.

8. Nicolas-Puel C, Faulconbridge RL, Guitton M, Puel JL, Mondain M, Uziel A. Characteristics of tinnitus and etiology of associated hearing loss: a study of 123 patients. Int Tinnitus J 2002;8:37-44.

9. Davis A, Rafaie EA. Epidemiology of tinnitus. In: Tyler RS, Editor. Tinnitus Handbook. New York: Cengage Learning, Inc, 2000, p.1-23.

10. Moller AR. The role of neural plasticity in tinnitus. Prog Brain Res 2007;
166:37-45.

11. Holgers KM, Zoger S, Svedlund K. Predictive factors for development of severe tinnitus suffering-further characterisation. Int J Audiol 2005; 44:584-592.

12. Milerova J, Anders M, Dvorak T, Sand PG, Koniger S, Langguth B. The influence of psychological factors on tinnitus severity. Gen Hosp Psychiatry 2013;35:412-416.

13. Meikle MB, Vernon J, Johnson RM. The perceived severity of tinnitus. Some observations concerning a large population of tinnitus clinic patients. Otolaryngol Head Neck Surg 1984;92:689-696.

14. Dobie RA. Depression and tinnitus. Otolaryngol Clin North Am 2003; 36:383-388.

15. Harrop-Griffiths J, Katon W, Dobie R, Sakai C, Russo J. Chronic tinnitus: association with psychiatric diagnoses. J Psychosom Res 1987;31: 613-621.

16. Sullivan MD, Katon W, Dobie R, Sakai C, Russo J, Harrop-Griffiths J. Disabling tinnitus. Association with affective disorder. Gen Hosp Psychiatry 1988;10:285-291.

17. Pinto PC, Marcelos CM, Mezzasalma MA, Osterne FJ, de Lima MDMT, Nardi AE. Tinnitus and its association with psychiatric disorders: systematic review. J Laryngol Otol 2014;128:660-664.

18. Tyler RS, Coelho C, Noble W. Tinnitus: standard of care, personality differences, genetic factors. ORL J Otorhinolaryngol Relat Spec 2006;68: 14-19; discussion 20-12.

19. Salviati M, Bersani FS, Terlizzi S, Melcore C, Panico R, Romano GF, et al. Tinnitus: clinical experience of the psychosomatic connection. Neuropsychiatr Dis Treat 2014;10:267-275.

20. Kim YH, Jung HJ, Kang SI, Park KT, Choi JS, Oh SH, et al. Tinnitus in children: association with stress and trait anxiety. Laryngoscope 2012; 122:2279-2284.

21. Oishi N, Shinden S, Kanzaki S, Saito H, Inoue Y, Ogawa K. Influence of depressive symptoms, state anxiety, and pure-tone thresholds on the tinnitus handicap inventory in Japan. Int J Audiol 2011;50:491-495.

22. Reiss S, Peterson RA, Gursky DM, McNally RJ. Anxiety sensitivity, anxiety frequency and the prediction of fearfulness. Behav Res Ther 1986; 24:1-8.

23. Andersson G, Vretblad P. Anxiety sensitivity in patients with chronic tinnitus. Scand J Behav Ther 2000;29:57-64.

24. Gul AI, Ozkiris M, Aydin R, Simsek G, Saydam L. Coexistence of anxiety sensitivity and psychiatric comorbidities in patients with chronic tinnitus. Neuropsychiatr Dis Treat 2015;11:413-418.

25. Newman CW, Jacobson GP, Spitzer JB. Development of the tinnitus handicap inventory. Arch Otolaryngol Head Neck Surg 1996;122:143148.

26. Kim JH, Lee SY, Kim CH, Lim SL, Shin JN, Chung WH, et al. Reliability and validity of a Korean adaptation of the tinnitus handicap inventory. Korean J Otorhinolaryngol-Head Neck Surg 2002;45:328-334.

27. Won H, Park H, Kwon S. A study on the development of the Korean versions of panic scales. Korean J Clin Psychol 1995;14:95-110.

28. Telch MJ, Shermis MD, Lucas JA. Anxiety sensitivity: unitary personality trait or domain-specific appraisals? J Anxiety Disord 1989;3:25-32.

29. Lilienfeld SO, Turner SM, Jacob RG. Anxiety sensitivity: an examination of theoretical and methodological issues. Adv Behav Res Ther 1993;15:147-183.

30. Zigmond AS, Snaith RP. The hospital anxiety and depression scale. Acta Psychiatr Scand 1983;67:361-370.

31. Oh SM, Min KJ, Park DB. A study on the standardization of the hospital anxiety and depression scale for Koreans: a comparison of normal, depressed and anxious groups. J Korean Neuropsychiatr Assoc 1999; 38:289-296.

32. Monzani D, Genovese E, Marrara A, Gherpelli C, Pingani L, Forghieri $\mathrm{M}$, et al. Validity of the Italian adaptation of the Tinnitus Handicap Inventory; focus on quality of life and psychological distress in tinnitussufferers. Acta Otorhinolaryngol Ital 2008;28:126-134.

33. Erlandsson SI, Hallberg LR. Prediction of quality of life in patients with 
tinnitus. Br J Audiol 2000;34:11-20.

34. Ghodratitoostani I, Zana Y, Delbem AC, Sani SS, Ekhtiari H, Sanchez TG. Theoretical tinnitus framework: a neurofunctional model. Front Neurosci 2016;10:370.

35. Zöger S, Svedlund J, Holgers KM. Relationship between tinnitus severity and psychiatric disorders. Psychosomatics 2006;47:282-288.

36. Spielberger CD. State-Trait Anxiety Inventory. New Jersey: John Wiley \& Sons, Inc; 2010
37. Hesser H, Andersson G. The role of anxiety sensitivity and behavioral avoidance in tinnitus disability. Int J Audiol 2009;48:295-299.

38. Andersson G. Psychological aspects of tinnitus and the application of cognitive-behavioral therapy. Clin Psychol Rev 2002;22:977-990.

39. Smits JAJ, Berry AC, Tart CD, Powers MB. The efficacy of cognitive-behavioral interventions for reducing anxiety sensitivity: a meta-analytic review. Behav Res Ther 2008;46:1047-1054. 